\title{
2016 Leroy P. Steele Prizes
}

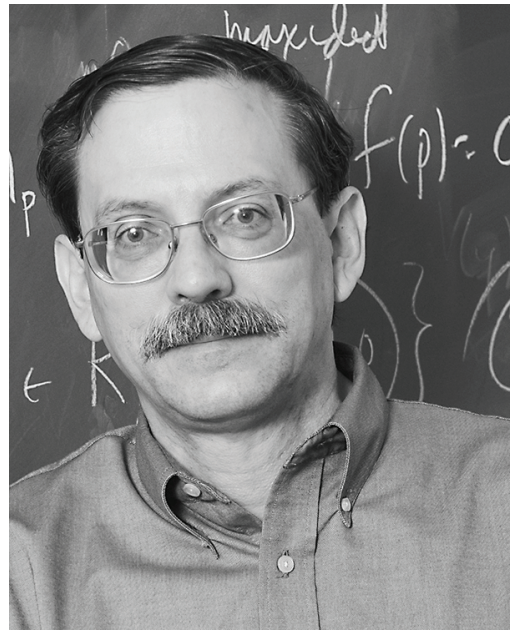

David Cox

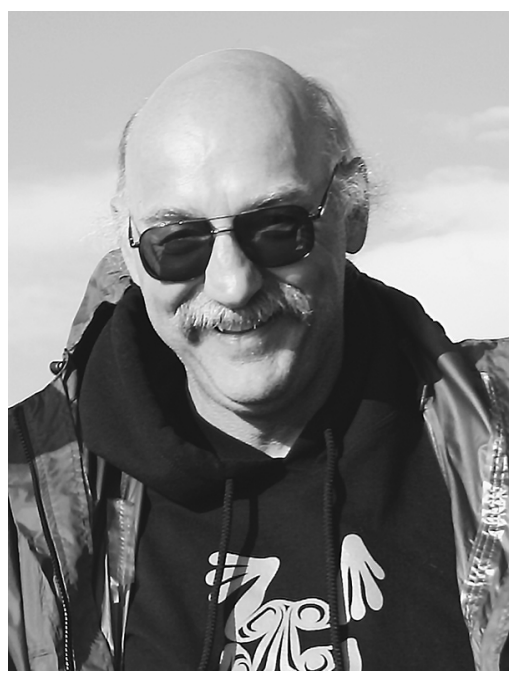

Andrew J. Majda

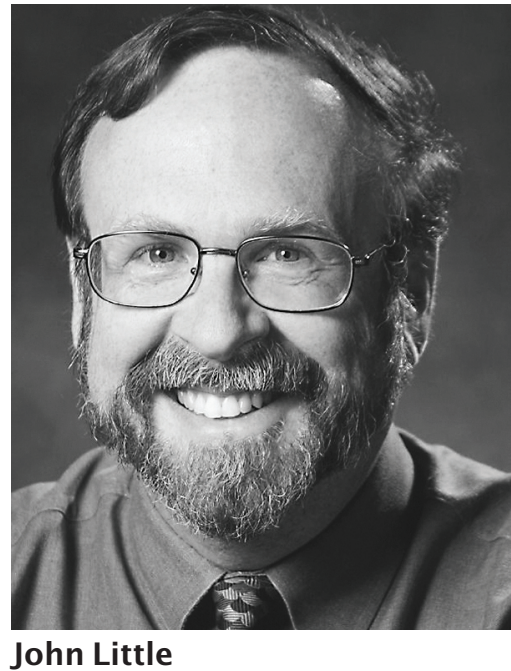

John Little

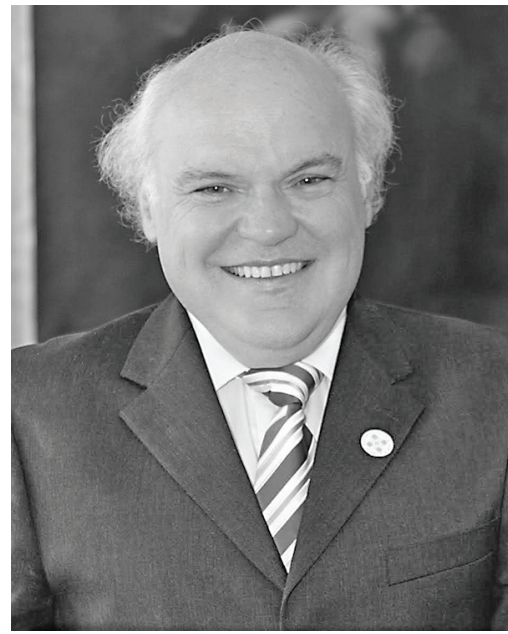

Donal O'Shea

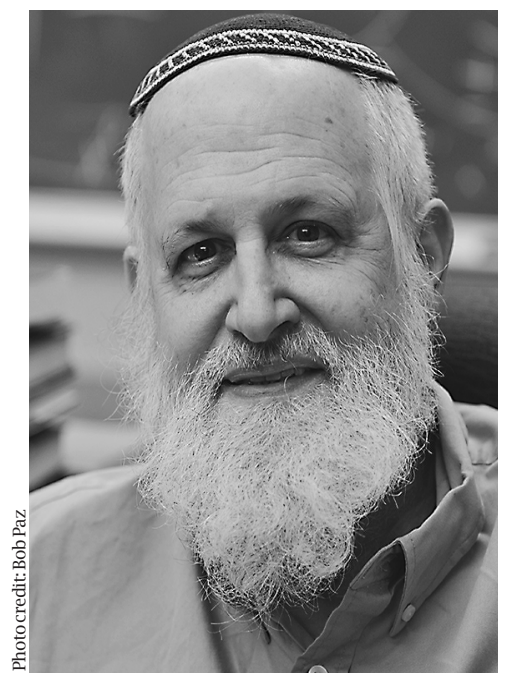

Barry Simon
The 2016 Leroy P. Steele Prizes were presented at the 122nd Annual Meeting of the AMS in Seattle, Washington, in January 2016. The Steele Prizes were awarded to DAVID COX, JohN LITTLE, and DONAL O'SHEA for Mathematical Exposition; to ANDREW J. MAJDA for Seminal Contribution to Research in Applied Mathematics; and to BARRY SIMON for Lifetime Achievement.

For permission to reprint this article, please contact: reprint-permission@ams.org.

DOI: http://dx.doi.org/10.1090/noti1357
Citation for Mathematical Exposition: David Cox, John Little, and Donal O'Shea

The 2016 Leroy P. Steele Prize for Mathematical Exposition is awarded to David Cox, John Little, and Donal O'Shea for their book Ideals, Varieties, and Algorithms [Undergraduate Texts in Mathematics, Springer-Verlag, New York, four editions 1992, 1997, 2007, 2015] that has made algebraic geometry and computational commutative algebra accessible not just to mathematicians but to students and researchers in many fields.

With the 1992 publication of Ideals, Varieties, and Algorithms, the authors, all then faculty at 
liberal arts colleges, invited a broad audience to study algebraic geometry by writing an undergraduate text with only linear algebra as a prerequisite. Throughout the book the authors maintain fidelity to their assumptions about their reader's background, using geometry to introduce core topics and appealing to computational theory to prove fundamental results. They complement the development of theoretical results with applications to such topics as automated theorem proving and robotics. Clear algorithms are provided that help the reader to further understand the concepts.

Even more impressive than its clarity of exposition is the impact it has had on mathematics. CLO, as it is fondly known, has not only introduced many to algebraic geometry, it has actually broadened how the subject could be taught and who could use it. One supporter of the nomination writes, "This book, more than any text in this field, has moved computational algebra and algebraic geometry into the mathematical mainstream. I, and others, have used it successfully as a text book for courses, an introductory text for summer programs, and a reference book."

Another writer, who first met the book in an REU two years before it was published, says, "Without this grounding, I would have never survived my first graduate course in algebraic geometry." This theme is echoed in many other accounts: "I first read CLO at the start of my second semester of graduate school.... Almost twenty years later I can still remember the relief after the first hour of reading. This was a math book you could actually read! It wasn't just easy to read but the material also grabbed me."

For those with a taste for statistics, we note that CLO has sold more than 20,000 copies, it has been cited more than 850 times in MathSciNet, and it has 5,000 citations recorded by Google Scholar. However, these numbers do not really tell the story. Ideals, Varieties, and Algorithms was chosen for the Leroy P. Steele Prize for Mathematical Exposition because it is a rare book that does it all. It is accessible to undergraduates. It has been a source of inspiration for thousands of students of all levels and backgrounds. Moreover, its presentation of the theory of Groebner bases has done more than any other book to popularize this topic, to show the powerful interaction of theory and computation in algebraic geometry, and to illustrate the utility of this theory as a tool in other sciences.

\section{Biographical Sketch of Cox}

David A. Cox went to Rice University and received his $\mathrm{PhD}$ from Princeton University in 1975 under the direction of Eric Friedlander. After teaching at Haverford College and Rutgers University, he moved to Amherst College in 1979, where he is now the William J. Walker Professor of Mathematics. He has served on the editorial boards of the Pure and Applied Mathematics series published by Wiley and the Graduate Studies in Mathematics series of the AMS, and he is currently on the board of the Undergraduate Texts in Mathematics series of Springer. He gave an AMS-MAA Joint Invited Address in 1995 and served on the Council of the AMS from 1991 to 1994. In 2012, he became a fellow of the AMS and received a Lester R. Ford Award from the MAA for his 2011 American Mathematical Monthly article, "Why Eisenstein proved the Eisenstein Criterion and why Schönemann discovered it first", 118, No. 1. Cox's current areas of research include toric varieties and the commutative algebra of curve and surface parametrizations, though he also has interests in number theory and the history of mathematics. He is the author of texts on number theory, computational algebraic geometry, mirror symmetry, Galois theory, and toric varieties. He loves teaching at all levels of the undergraduate curriculum and greatly enjoys working with students on senior theses. He has interacted with graduate students in numerous summer programs, including MSRI Summer Graduate Workshops in Berkeley and Cortona, Italy.

\section{Biographical Sketch of Little}

John Little earned an AB from Haverford College in 1976 and a $\mathrm{PhD}$ in mathematics from Yale University in 1980 under the direction of Bernard Saint-Donat. He has taught at the College of the Holy Cross since 1980 and is currently professor of mathematics. He has supervised many undergraduate research projects, including work at eight REU-type summer programs - at Amherst College, at Mount Holyoke College, at two campuses of the University of Puerto Rico, at MSRI in Berkeley, and at the University of Hawaii at Hilo. Starting in the mid-1980s he developed a strong interest in computational techniques in algebraic geometry and commutative algebra that led to Ideals, Varieties, and Algorithms and its companion volume Using Algebraic Geometry. In recent research work, he has considered applications of computational methods in algebraic geometry to areas such as error-control coding theory, signal processing, and celestial mechanics.

\section{Biographical Sketch of O'Shea}

Donal O'Shea has been president and professor of mathematics at New College of Florida since 2012. Prior to that, he was at Mount Holyoke College, the final fourteen years as vice president of academic affairs and dean of faculty. He received his undergraduate degree from Harvard University and his $\mathrm{PhD}$ from Queen's University in Canada under the direction of A. John Coleman. He has spent extended research leaves at IHES, IHP, and the Universities of Amherst, Kaiserslautern, Hawaii, Miami, Edinburgh, and Cambridge. His favorite research interests concern the topological and geometric (most recently, bi-Lipschitz) behavior of hypersurface singularities. He enjoys teaching, reading, writing, cooking (which he unfortunately 
eats too much of), and shares life with his wife and best friend, Mary, their four grown children, Seamus, Brendan, Sarah, and Kathleen, their partners, and his five, soon to be six, grandchildren.

\section{Joint Response from David Cox, John Little, and Donal O'Shea}

We are deeply honored and grateful to receive the 2016 Leroy P. Steele Prize for Mathematical Exposition for our book Ideals, Varieties, and Algorithms. We started writing it in 1988, partly to provide background materials and notes for students involved in undergraduate research, and partly to provide alternatives to the usual algebra courses for undergraduate mathematics majors. At that time, new algorithms and ever-faster computing speeds were making it possible for undergraduates to explore examples that would previously have been inaccessible without deeper theoretical tools, a trend which has only increased in the intervening years. Through four editions,we have learned a tremendous amount by working on this project, and our readers include many individuals who expanded our horizons and whom we would never have encountered otherwise.

Many wonderful books and papers in mathematics have appeared in the last few decades, and those singled out by the Leroy P. Steele Prize for Mathematical Exposition are among the best. Having our book listed in this company gives us great pleasure, and we are deeply touched by the citation. We worked hard to keep the book accessible to those with a modest background in mathematics, especially undergraduates, while including enough novel topics and new points of view to interest experts in the subject. The avoidance of abrupt changes in mathematical sophistication was almost as challenging as presenting algebraic geometry without using the full power of its modern abstract language. Although writing for students, we were surprised and gratified by the delight which professional mathematicians and other scientists and engineers have taken in the book. Writing for undergraduates is not always a recipe for elegance, but just as universal design principles seem to result in better buildings, our focus on accessibility resulted in a better and more usable book.

We thank the generations of readers who have sent us suggestions and pointed out infelicities and errors. We thank our colleagues who read successive drafts, and our families and friends from whom we took the time to prepare them. We thank those who nominated the book. And we thank the American Mathematical Society for honoring a book that was written to be accessible to undergraduates. When learning of the award, one of us wrote the others saying that "collaborating with the two of you has been one of the greatest pleasures of my professional life." He voiced what we each felt, and although we like to think that we would have eventually told each other this, we are grateful that the award nudged us into full disclosure. And we thank you, the members of the extended mathematical community, for the encouragement and collegiality that has led to this great honor. We are in your debt.

\section{Citation for Seminal Contribution to Research in} Applied Mathematics: Andrew J. Majda

Andrew J. Majda, the Samuel F. B. Morse Professor of Arts and Sciences at the Courant Institute of Mathematical Sciences at New York University, is awarded the 2016 Leroy P. Steele Prize for Seminal Contribution to Research in Applied Mathematics for two papers published in the Memoirs of the American Mathematical Society in 1983: "The existence of multidimensional shock fronts", 43, No. 28 , and "The stability of multidimensional shock fronts" 41, No. 27.

Multidimensional systems of conservation laws are important models in fluid mechanics in the absence of dissipation. The mathematical difficulty in the analysis of these partial differential equations is the development of singularities and the appearance of shocks. Riemann recognized that an important step in the study of conservation laws is the understanding of the structure of the shock fronts/waves, and he considered the problem in one space-dimension.

This is where the work of Majda begins with emphasis on the multidimensional setting. The cited works, which shortly after appeared also in the book Compressible Fluid Flow and Systems of Conservation Laws in Several Space Variables [Applied Mathematical Sciences, 53, Springer-Verlag, New York, 1984] are pioneering and seminal. They were written at a time when research on hyperbolic systems of conservation laws was extremely active but focused on the one-dimensional case or spherically symmetric solutions. The aforementioned papers pioneered the expansion to the multidimensional case by providing the first rigorous treatment of the existence and stability of multidimensional shock fronts. This required very detailed and hard analysis, and it is fair to say that the papers are a tour de force. The analysis revealed a number of new instability phenomena that are not present in the one-space-dimensional case. This work, which immediately became classic, is at the same level of importance as the entropy solutions of Lax and the random choice method of Glimm.

\section{Biographical Sketch of Majda}

Andrew J. Majda is the Samuel F. B. Morse Professor of Arts and Sciences at the Courant Institute of Mathematical Sciences at New York University. He was born in East Chicago, Indiana, on January 30, 1949. He received a BS degree from Purdue University in 1970 and a PhD degree from Stanford University in 1973. Majda began his scientific career as a Courant Instructor at the Courant Institute from 1973-75. Prior to returning to the 
Courant Institute in 1994, he held professorships at Princeton University (1984-94), the University of California Berkeley (1978-84), and the University of California Los Angeles (1976-78).

Majda's primary research interests are modern applied mathematics in the broadest possible sense merging asymptotic methods, numerical methods, physical reasoning, and rigorous mathematical analysis. He is well known for both his theoretical contributions to partial differential equations and his applied contributions to diverse areas such as scattering theory, shock waves, combustion, incompressible flow, vortex motion, turbulent diffusion, and atmosphere ocean science.

Majda is a member of the National Academy of Sciences and the American Academy of Arts and Sciences, and he has received numerous honors and awards, including the National Academy of Science Prize in Applied Mathematics, the John von Neumann Prize of the Society of Industrial and Applied Mathematics, and the Gibbs Prize of the American Mathematical Society. In 2012 he received the Norbert Wiener Prize of the American Mathematical Society and the Society of Industrial and Applied Mathematics, and in 2015 Majda received the Lagrange Prize, awarded every four years by the International Council of Industrial and Applied Mathematics. He has been awarded the Medal of the College de France twice and is a fellow of the Japan Society for the Promotion of Science. Majda has received three honorary doctorates, including one from his undergraduate alma mater, Purdue University. He has given plenary one-hour lectures at both the ICM (Kyoto 1990) and the first ICIAM (Paris 1987) and is a fellow of both the AMS and SIAM.

In his years at the Courant Institute, Majda has created the Center for Atmosphere Ocean Science with seven multidisciplinary faculty members to promote cross-disciplinary research with modern applied mathematics in climate modeling and prediction.

\section{Response from Andrew J. Majda}

I am honored and pleased to receive the 2016 Leroy P. Steele Prize for Seminal Contributions to Research in Applied Mathematics. My work cited on multidimensional shock fronts is part of the mathematically beautiful yet practical area of hyperbolic conservation laws such as, for example, the flow of gases or water at both low and high speeds. The serendipity between mathematical structure and physical phenomena attracted me to this research topic. I would like to acknowledge many members of the Courant Institute who strongly influenced my interest in shock waves when I was a young postdoc, including Joe Keller, Cathleen Morawetz, and especially Peter Lax, as well as the book by Courant and Friedrichs on supersonic flow.
Citation for Lifetime Achievement: Barry Simon

The 2016 Leroy P. Steele Prize for Lifetime Achievement is awarded to Barry Simon, the IBM Professor of Mathematics and Theoretical Physics at the California Institute of Technology, for his tremendous impact on the education and research of a whole generation of mathematical scientists. His significant research achievements, highly influential books, and mentoring of graduate students and postdocs comprise a career of exceptional achievement.

His accomplishments span 333 papers and sixteen books on topics ranging from mathematical physics to orthogonal polynomials. Simon proved a number of fundamental results in statistical mechanics, the most significant of which is the proof of phase transitions for the classical Heisenberg ferromagnet and the quantum antiferromagnet. Almost forty years later these are the only rigorous proofs of continuous symmetry breaking with a non-Abelian symmetry group.

Simon was an important contributor to the construction of quantum fields in two space-time dimensions. His work with Guerra and Rosen, especially their 146-page paper published in the Annals of Mathematics in 1975, established an analogy with classical statistical mechanics which led to a number of new insights. An outgrowth of his work in this area is the theory of hypercontractive semigroups, which in turn led to a theory of ultracontractivity.

These topics, which have grown into major industries, figure prominently in Simon's work on the general theory of Schrödinger operators, where he proved a number of definitive results. In this effort he was a pioneer in the use of path integral techniques, which he beautifully described in his book Functional Integration and Quantum Mechanics [Pure and Applied Mathematics, 86, Academic Press, Inc., New York-London, 1979]. In the past fifteen years, he has advanced the theory of orthogonal polynomials, elucidating and expanding upon many apsects of Szegö's theorem. His work on this topic, with Killip Rowan and David Damanik, includes two papers in Annals of Mathematics and one in Inventiones Mathematicae.

His four-volume set of books written with Mike Reed, Methods of Modern Mathematical Physics [Academic Press, Inc., New York-London, 1972, 1975, 1979, 1978], "played the role that Courant and Hilbert I and II did for the preceding generation. This is true not only in the West; the Russian translation was a bible for a generation of analysts and mathematical physicists behind the Iron Curtain," to quote the nominators. Similarly, his two-volume set of AMS Colloquium Publications, Orthogonal Polynomials on the Unit Circle [American Mathematical Society, Providence, RI, 2005] became instant classics connecting the theory of orthogonal polynomials with the spectral 
theory of Schrödinger operators and other topics in mathematical physics. In 2015, Simon won the International János Bolyai Prize of Mathematics for these books.

\section{Biographical Sketch of Simon}

Barry Simon is the IBM Professor of Mathematics and Theoretical Physics at the California Institute of Technology. He was born in Brooklyn, New York, in 1946, received a BA from Harvard University (where he was a top-five Putnam winner) in 1966, and a $\mathrm{PhD}$ in physics from Princeton University in 1970. He started as an instructor in mathematics at Princeton in 1969-70, but since then all his appointments at Princeton and Caltech have been joint. He received tenure in 1972, two years after Charlie Fefferman, who was in the same graduate class. Since 1981, he has been at Caltech, where he served as executive officer (chair) for ten years. Simon has published just under 400 papers and twenty-one math books (plus several end-user-oriented computer books). His most recent books are the five-volume (3,200 pages) Comprehensive Course in Analysis published by the AMS in December 2015. At Google Scholar his citation number is about 60,000 , and his $h$-index is 102 (i.e., 102 publications with at least 102 citations). His research has been in many aspects of mathematical physics and in the analytic theory of orthogonal polynomials.

He has been recognized with honorary degrees from Technion, the University of Wales-Swansea, and LMU-Munich. He was awarded the Poincaré Prize of the IAMP in 2012 and the Bolyai Prize of the Hungarian Academy of Science in 2015. He is a fellow of the American Physical Society (1981), the American Academy of Arts and Sciences (2005), and the AMS (2013). He has served as vice president of the AMS and of IAMP.

\section{Response from Barry Simon}

I should like to thank the AMS and especially the Leroy P. Steele Prize Committee for this noteworthy recognition. It is a great honor to join the distinguished list of former winners. I'd also like to acknowledge my mentors Arthur Wightman and Ed Nelson, who have passed away in the past few years. They not only taught me mathematics but how to be a mathematician.

I am especially pleased by this prize since it recognizes not only my research but my greater impacts. At the perhaps silly level this includes a listing of terms which appeared first in my papers, including hypercontractive and ultracontractive semigroups, Birman-Schwinger principle, diamagnetic inequality, Kato's inequality, CLR bound, Berry's phase, almost Mathieu equation, wonderland theorem, OPUC/OPRL, Verblunsky coefficients, CMV matrix, and clock behavior.

More importantly, it recognizes the impact of my books. It is always a little thrill when I get into an email discussion with someone who then mentions that it was Reed and Simon that first turned them on to functional analysis. I take pleasure in the invigoration of the analytic theory of OPUC that was caused in part by those books. And I hope that my most recent five-volume opus will have impact.

Most directly, I cherish the impact on thesis students, postdocs, and others I've mentored. Mathematics is a communal enterprise, and I've taken great joy in my interaction with coauthors and those I've taught.

\section{About the Prizes}

The Leroy P. Steele Prizes were established in 1970 in honor of George David Birkhoff, William Fogg Osgood, and William Caspar Graustein. Osgood was president of the AMS during 1905-06, and Birkhoff served in that capacity during 1925-26. The prizes are endowed under the terms of a bequest from Leroy P. Steele. Up to three prizes are awarded each year in the following categories: (1) Lifetime Achievement for the cumulative influence of the total mathematical work of the recipient, high level of research over a period of time, particular influence on the development of a field, and influence on mathematics through PhD students; (2) Mathematical Exposition for a book or substantial survey or expository research paper; (3) Seminal Contribution to Research for a paper, whether recent or not, that has proved to be of fundamental or lasting importance in its field or a model of important research. The Steele Prizes for Mathematical Exposition and Seminal Contribution to Research carry a cash award of US \$5,000; the Prize for Lifetime Achievement, a cash award of $\$ 10,000$.

The list of previous recipients of the Steele Prizes may be found on the AMS website at Www. ams.org/prizes-awards.

The members of the Committee to Select the Winners of the Leroy P. Steele Prizes for 2016 were the following individuals.

- Paul F. Baum

- Richard T. Durrett (Chair)

- Phillip Griffiths

- Nancy J. Kopell

- Jeffrey C. Lagarias

- Nikolai Makarov

- Vladimir Markovic

- Andrei Okounkov

- Yuval Peres

- Karen Uhlenbeck

The Prize for Seminal Contribution to Research is awarded on a six-year cycle of subject areas. The 2016 prize was given in applied mathematics. The 2017 prize will be given in geometry/topology; the 2018 prize in discrete mathematics/logic; the 2019 prize is open; the 2020 prize in analysis/probability; and the 2021 prize in algebra/number theory. 